\title{
INVESTIGACIÓN/RESEARCH
}

Recibido: 28/10/2013-----Aceptado: 14/01/2014-----Publicado: 15/03/2014

\section{EROTISMO Y SEXUALIDAD EN TRES PELÍCULAS DIRIGIDAS POR STEVEN SPIELBERG EN LAS ÚLTIMAS TRES DÉCADAS DEL SIGLO $\mathbf{X X}$}

José Díaz-Cuesta': CILAP, Universidad de La Rioja. España.

jose.diaz-cuesta@unirioja.es

\section{RESUMEN:}

El presente artículo se acerca al tratamiento de la sexualidad y el erotismo en tres obras de Steven Spielberg: Close Encounters of the Third Kind, The Color Purple $y$ Schindler's List, cada una de ellas perteneciente a una de las tres últimas décadas del siglo XX. Tras recorrer los tres textos fílmicos buscando referencias explícitas o veladas a ambos conceptos, se llega a la conclusión de que es posible observar una evolución en el tratamiento que Spielberg les da con el paso del tiempo. Así, en Close Encounters of the Third Kind nos encontramos con besos y con una trama paralela a la principal que refleja el aproximamiento entre un hombre y una mujer. The Color Purple sitúa el abuso sexual en el centro de la narración, sin llegar a mostrarlo explícitamente, y tratando con mucha delicadeza el lesbianismo. En Schindler's List es posible observar al protagonista realizando el acto sexual con una de sus amantes. Pero esto son solo tres muestras de la filmografía de Spielberg: los resultados obtenidos incitan a seguir indagando en este campo en el resto de su obra.

PALABRAS CLAVE: Steven Spielberg - Sexualidad - Erotismo - Encuentros en la tercera fase - El color púrpura - La lista de Schindler

\footnotetext{
1 José Díaz Cuesta: Profesor Contratado Doctor de la Facultad de Letras y Educación de la Universidad de La Rioja. España.

Correo: jose.diaz-cuesta@unirioja.es
} 


\title{
EROTISM AND SEXUALITY IN THREE FILMS DIRECTED BY STEVEN SPIELBERG IN THE LAST THREE DECADES OF THE TWENTIETH CENTURY
}

\begin{abstract}
:
This journal article deals with the treatmeant of sexuality and erotism in three films directed by Steven Spielberg: Close Encounters of the Third Kind, The Color Purple and Schindler's List. Each of these films belongs to one of the last decades of the Twentieth century. After going through the three film texts in search of explicit or implicit references to both concepts, it can be concluded that it is possible to observe an evolution in Spielberg's treatment with the passing of time. In Close Encounters of the Third Kind we find kisses and a subplot which mirrors the approach between a man and a woman. The Color Purple places sexual abuse at the centre of the narrative, without showing it explicityly, and dealing with lesbianism in a very delicate way. In Schindler's List we can watch the protagonist making love with one of his lovers. But these are only samples of Spielberg's filmography: the results we have reached invite us to keep on exploring these concepts throughout the whole of his work.
\end{abstract}

KEY WORDS: Spielberg - Sexuality - Erotism - Close Encounters of the Third Kind The Color Purple - Schindler's List.

\section{INTRODUCCIÓN ${ }^{2}$}

El tema que nos hemos propuesto abordar en este artículo, el de la sexualidad y el erotismo en tres películas dirigidas por Steven Spielberg en el siglo XX, puede que sorprenda a más de una persona que se acerque a estas páginas, pero esperamos que tras la lectura de las mismas la idea de relacionar ambos conceptos con la obra de Spielberg no parezca descabellada.

Las monografías académicas dedicadas a la obra de Steven Spielberg apenas si se han fijado en este componente de su filmografía. En inglés encontramos las obras de Yosefa Loshitzky (1997), Charles Silet (2002), Warren Buckland (2006, Lester Friedman (2006), Nigel Morris (2007), Andrew Gordon (2008), Dean Kowalski (2008) y Frederick Wasser (2010). En español, las de Antonio Sánchez-Escalonilla (1995 y 2004) y David Caldevilla (2005). Nosotros tampoco hemos prestado especial atención a este aspecto al abordar el entorno del mundo externo de los protagonistas masculinos en las cinco obras de monstruos que Spielberg ha dirigido hasta la fecha actual (Díaz Cuesta, 2010), centrándonos más en la importancia del rol paterno3.

\footnotetext{
${ }^{2}$ Parte de la investigación para este artículo fue financiada por el proyecto de la Universidad de la Rioja denominado GENEID, de referencia PROFAI13/21, subvencionado por el Banco Santander.

${ }^{3}$ Estos resultados se hacen más patentes en Díaz-Cuesta (2012).
} 
Steven Spielberg - Sexualidad - Erotismo - Encuentros en la tercera fase - El color púrpura - La lista de Schindler

Las obras que hemos escogido son, por orden de estreno, Close Encounters of the Third Kind (1977), The Color Purple (1985) y Schindler's List (1993). Varias son las razones por las que hemos escogido precisamente estas películas.

En el caso de la primera, Close Encounters of the Third Kind, consideramos que pertenece al grupo de películas fantásticas - por darles una definición no demasiado comprometida- de Spielberg: Friedman (2006, pp. 11-62) la incluye dentro de la categoría de películas fantásticas y de ciencia ficción.

La segunda, The Color Purple, supone un cambio en la línea de acción del director, ya que opta decididamente por intentar realizar un cine melodramático que vaya dirigido a un público más adulto, a diferencia del para todos los públicos de sus obras anteriores. Friedman (p. 244) la engloba dentro de la categoría de películas sobre minorías étnicas y problemas sociales.

La última película que explorams, Schindler's List, constituye la obra maestra del director, o al menos una de las más originales, ya que con ella Spielberg crea un estilo nuevo a la hora de narrar. Para Friedman (p. 290), se encuadra dentro de la categoría de películas sobre el Holocausto.

Por otro lado, Close Encounters of the Third Kind está construida sobre un guion del propio director, que es el único en aparecer en los títulos de crédito como guionista, aunque sí hubiera otros co-guionistas que colaboraron con él en este aspecto, según aclara John Baxter (1996, pp. 154-155) en la biografía, no autorizada, Steven Spielberg. El guion de The Color Purple es obra de Menno Meyjes, en una traducción directa de la novela homónima escrita por Alice Walker (1982) ${ }^{4}$. Schindler's List también se basa en una novela de igual título, obra del escritor australiano Thomas Keneally (1982), que se limita a dar forma de novela a una serie de hechos reales y documentados $^{5}$, la mayor parte de los cuales se mantienen en el guion de Steven Zaillian. De esta forma podemos observar que las tres películas tienen orígenes muy diferentes: una idea original en el primer caso, una novela en el segundo y hechos reales - novelados- en el tercero.

Como última razón para nuestra elección hemos de señalar que entre el estreno de una película y la siguiente transcurren ocho años, tiempo más que suficiente como para que un creador tan prolífico como Spielberg experimente cambios, y que cada una de ellas apareció en las pantallas de todo el mundo en décadas diferentes. A pesar de todas las razones que hemos aducido, y algunas otras que se nos hayan quedado en el tintero, no puedo dejar de recordar la idea de Jesús González Requena de que no es el o la analista quien elige los textos que comenta, sino que son estos los que escogen a él o a ella.

\footnotetext{
${ }^{4}$ Para una comparación entre novela y película, véase Digby (1993) y Díaz-Cuesta (1999).

${ }^{5}$ Para una comparación entre ambas obras, véase Díaz-Cuesta (2003), y para una exploración por varios autores de las diferentes relaciones entre ellas, véase Fensch (1995).
} 
A pesar de la brevedad de nuestro corpus, creemos que las películas elegidas pueden considerarse representativas de gran parte del abanico de posibilidades que la obra spielberguiana presenta. Todas ellas guardan una cierta relación con realidades iberoamericanas: el avistamiento de ovnis es un fenómeno global, y así se refleja en las primeras escenas de Close Encounters of the Third Kind, en las que algunas de las primeras palabras que se pronuncian están en español; el machismo retratado en The Color Purple, con otros sesgos, es lamentablemente moneda común en la comunidad iberoamericana; y por último Argentina, en la vida real, fue refugio del matrimonio Schindler representado en Schindler's List, al igual que acogió a algunos otros nazis, así como a judíos supervivientes del Holocausto.

\section{OBJETIVOS}

Con este artículo se pretende demostrar que temas como el erotismo y la sexualidad no son ajenos al cine de Steven Spielberg. Además, gracias a la inclusión de tres películas de décadas diferentes en nuestro corpus, podemos observar qué grado de evolución se produce en el tratamiento de esos temas a lo largo de dieciséis años de la carrera cinematográfica de este director. Por último se pretende descubrir si los resultados obtenidos tienen la suficiente repercusión como para seguir investigando estos temas en el resto de la filmografía spielberguiana.

\section{METODOLOGÍA}

El método que hemos seguido en nuestra exploración es analítico-sintético. El análisis realizado es cercano, centrado en los aspectos bajo escrutinio, y se acerca al textual, sin llegar al nivel de detalle de este último. En las conclusiones se sintetizan los análisis realizados, y se ponen en valor.

\section{RESULTADOS}

Pasemos ahora a analizar cada una de las películas cronológicamente por separado, para al final ver qué podemos concluir en general de todas ellas.

\subsection{Close Encounters of the Third Kind}

Aunque el título de la película aluda a la tercera fase de encuentro con vida extraterrestre, esta fase no se produce hasta los momentos finales, y todo lo anterior del film se dedica a mostrar las otras dos fases del encuentro. La primera de ellas la constituye el avistamiento de un ovni (objeto volador no identificado) y la segunda la evidencia física que esos objetos dejen de su existencia. La tercera se produce si se llega al contacto físico con los ocupantes de un ovni. No resulta difícil establecer un paralelismo entre este tipo de encuentros, cuya expresión inglesa es, traducida literalmente, la de encuentros cercanos (close encounters), y el proceso de enamoramiento, o al menos de atracción física, entre dos personas. Pero no hará falta que dejemos volar la imaginación y bastará con que nos fijemos en lo que se cuenta en la propia película para darnos cuenta de la existencia de semejante paralelismo. 
La película narra el avistamiento inicial de varios ovnis por parte de Roy Neary (Richard Dreyfuss), a quien se ha presentado como un padre de familia con problemas de comunicación, tanto con su esposa como con sus hijos. El tema de la falta de comunicación, o más bien de la dificultad a la hora de comunicarse, es otro de las claves del film: en la secuencia inicial, a la que nos hemos referido anteriormente, vemos a varias personas hablando en inglés, español y francés con problemas a la hora de comunicarse; durante toda la película Claude LeCombe, el científico interpretado por Francois Truffaut, ha de enfrentarse a su condición de extranjero que no domina del todo la lengua del país que visita; finalmente humanos y alienígenas logran comunicarse gracias a un sistema combinado de luces notas musicales. Dreyfuss comparte este problema y, al igual que los demás, intentará darle solución. Justo después de haber avistado naves espaciales por primera vez, Neary se encuentra, también por vez primera, con Jillian Guiler (Melinda Dillon), cuyo hijo casi atropella. Guiler ya había sido presentada en la película en su papel de madre, rasgo por el que se la seguirá identificando a lo largo del film.

La evidencia física de la existencia de ovnis en la película podríamos ejemplificarla en los destrozos causados por su presencia, o incluso por las quemaduras que se producen en la piel de quien los ha visto. Roy y Jillian comparten esas quemaduras, hecho que ella le hace ver la próxima vez que se encuentran. Ese encuentro se produce junto a un gran teleobjetivo que bien puede leerse como símbolo fálico, premonición de otro símbolo que concentra la capacidad de deseo en la película. Me refiero a la Torre del diablo, la montaña que obsesiona a Roy y a Jillian incluso antes de saber de su existencia. La primera evidencia que ambos tienen de ella se produce cuando los dos tocan una reproducción en miniatura hecha por Barry, el hijo de Jillian. Más tarde, cuando Barry ya ha desaparecido, aparentemente secuestrado por un ovni, Roy y Jillian seguirán realizando reproducciones de la montaña, él en forma de esculturas cada vez de mayores proporciones hechas en materiales diversos - desde espuma de afeitar hasta barro, pasando por puré de patata-, ella en forma de pinturas y dibujos. La obsesión por la montaña, lejos de desaparecer, aumenta cuando ambos la ven por televisón: la reacción es la misma en los dos casos, pues tanto el uno como la otra tocan las pantallas de televisor de sus respectivas casas. La forma de la Montaña del diablo nos remite directamente al monolito de 2001: A Space Odyssey (Kubrick, 1968), al que se han dado un sinfín de interpretaciones. En el caso de la película que nos ocupa podemos hablar de una evolución en la forma, ya que lo primero que Neary esculpe presenta una forma marcadamente fálica que será transmutada por otra más uterina cuando secciona la parte superior con la intención de destruir la escultura por completo. Así, el hombre transforma el objeto de deseo de la mujer en el suyo propio, pero sin que el icono pierda todas sus connotaciones fálicas, ya que la forma no es descaradamente uterina, aunque sí lo suficiente para sugerir la idea de fertilidad. Nos encontramos así con un objeto de deseo bipolar que sirve de nexo de unión entre Roy y Jillian. Esta aparición del deseo se produce mientras se va deteriorando la relación de Roy con su mujer, quien le llega a negar violentamente el abrazo que su marido le pide la noche antes de abandonarlo llevándose a sus hijos, es decir, le niega un encuentro en la tercera fase. 
El contacto con extraterrestes, que no sucede hasta la conclusión del film, es prolépticamente anticipado por el contacto entre Roy y Jillian, que ocurre bastante antes, cuando se encuentran entre la masa de gente que está siendo evacuada, con engaños, de la zona del encuentro final. El montaje de esta secuencia entre Roy y Jillian, con planos en paralelo de uno y del otro, y el abrazo final que se dan, claramente se asemeja al que solemos ver en películas melodramáticas. La ascensión a la Torre del diablo la realizan cogidos de la mano, y finalmente, cuando Roy se dirige hacia la nave nodriza, se despiden con un beso en la boca. En esta última parte de la película también se puede observar un plano sostenido de la Torre, ya de noche, rodeada de estrellas, de puntos brillantes, algunos de los cuales pueden ser naves, que en ocasiones caen hacia la montaña, como si ésta fuera inseminada por el firmamento. Fruto de ese encuentro entre cielo y tierra nacerá el contacto entre humanos y alienígenas, que se ve personificado en la figura de Roy Neary, a quien permiten llevar a cabo otro encuentro en la tercera fase, pero esta vez con seres de otro planeta.

\subsection{The Color Purple}

El título de este texto fílmico alude al color malva que vemos varias veces en pantalla, principalmente en campos llenos de flores, cuya belleza alaba Shug Avery (Margaret Avery), pero también, sobre todo al final del film, en la ropa que lleva puesta la protagonista, Celie (Whoopi Goldberg), y su hermana Nettie, con la que por fin se reencuentra después de muchos años de separación. De nuevo otro film dirigido por Spielberg acaba con un encuentro en la tercera fase, si bien entre hermanas. Además en este caso se trata de un reencuentro, ya que lo que ha sucedido es que el marido de Celie las separa cuando ambas hermanas todavía son muy pequeñas, aunque ya no sean niñas. La tensión sensual se sitúa en la raíz de la separación, ya que Albert (Danny Glover) expulsa a Nettie de la casa en la que vive con Celie, su segunda mujer, que no es la madre de sus hijos. Albert no puede soportar el tener en su casa a una mujer que no puede, o, más bien, no logra poseer. La escena de mayor tensión sexual entre Albert y Nettie sucede cuando ésta va camino de la escuela y él la sigue a caballo. Los caballos constituyen una de los elementos masculinos de la película, a la manera de los westerns, y aquí Albert utiliza el vigor y la fuerza que estos animales representan para impresionar a Nettie, quien se defiende golpeándole, como se suele decir, donde más duele.

La tensión sexual no se limita a esta escena, sino que la película se encuentra plagada de ella. No debemos olvidar que la novela en que se basa constituye un claro alegato feminista que aquí se pretende reflejar lo más fielmente posible.

Si nos remontamos al comienzo del film, cuando se nos presenta a Celie, nos daremos cuenta de que desde un principio se intentan mostrar las consecuencias de la dominación de las mujeres por parte de los hombres.

En la primera secuencia, mientras todavía están apareciendo los títulos de crédito, vemos a dos niñas jugando. Para nuestra sorpresa, al oír la voz del supuesto padre de Celie, descubrimos que ésta se encuentra embarazada y que la criatura que lleva en 
Steven Spielberg - Sexualidad - Erotismo - Encuentros en la tercera fase - El color púrpura - La lista de Schindler

su vientre es fruto de las varias ocasiones en que aquél la ha violado. A pesar de ello el padre no desea a Celie sino a Nettie, al igual que Albert, quien acude - a caballo-, en su primera intervención en el film, a pedir la mano de la menor de las hermanas para acabar casándose con la mayor de las dos, pues el padre se niega a desprenderse de la hija que todavía no ha poseído. Es ese temor el que conduce a Nettie a la casa de Albert, pero allí, como ya hemos señalado, la situación aún se vuelve peor.

La película, como la novela, pretende ir más allá de la mera denuncia, ya que desde ella se proponen soluciones a la opresión masculina. Una de ellas, la más obvia, es la separación, algo que también sucede en la película, aunque nos interese más otro hecho que sucede antes y que no es otro que el de mantener relaciones sexuales con otra mujer. Esto se ve en la relación entre Celie y Shug Avery: Shug, amante de Albert, quien está perdidamente enamorado de ella, acaba siendo también la amante de Celie, hecho este último que se sugiere veladamente en la película. La primera reacción de Celie cuando descubre que la amante de su marido viene a vivir a su casa no es, ni mucho menos, la de una mujer celosa. Antes al contrario, la preocupación de Celie se centra en su aspecto físico, que intenta paliar de algún modo, pero sin lograr que lo primero que haga Shug al verla sea recordarle lo fea que es. La relación entre Celie y Shug constituye, a mi juicio, la parte más entrañable y mejor tratada de la película. Resulta destacable la escena en la que Celie se muestra completamente avergonzada por tener que asistir a Shug cuando se está dando un baño, ya que, claro está, esta última se encuentra desnuda. La atracción no ocurre solo en ese sentido, ya que Shug acaba correspondiendo ese amor que Celie siente por ella, primero con una canción que le dedica, significamente titulada Sister (Hermana), más tarde tocando y enseñando a besar a Celie, en una escena desarrollada por Spielberg con gran delicadeza, y por último llevándosela de la casa de Albert para acabar viviendo en la casa que la propia Celie ha heredado del hombre que creía era su padre.

Habría muchas más escenas que señalar, e incluso otras relaciones, como la de Sofia (Oprah Winfrey) con Harpo (Willard E. Pugh), el hijo de Albert, pero creo que con las que hemos analizado nos podemos dar cuenta de que en The Color Purple el erotismo y las relaciones sexuales constituyen uno de los temas principales de la película.

\subsection{Schindler's List}

La historia de Schindler's List es la de una pasión sin explicar: la que siente Oskar Schindler (Liam Neeson) por los judíos que acoge primero en su fábrica y más tarde en su propio y atípico campo de concentración.

Pero no es ésta la única pasión que alberga el industrial nazi. Entre otras, destacan su gusto por el dinero, la comida y las mujeres. Concentrémonos en esta última. Tanto la persona que fue Oskar Schindler como el personaje que aparece tanto en la novela como en la película muestran una gran afición por las mujeres. Sin embargo, a pesar de ese afán, Schindler no se distrae de su misión salvadora. En la película este hecho se muestra ya en la primera secuencia en la que vemos a Schindler con más gente. 
Schindler dirige su mirada hacia unas mujeres, mirada que éstas le devuelven sin que él les corresponda, ya que su atención se ha desviado hacia una mesa ocupada con militares nazis, que son el objetivo de su visita al restaurante. A pesar de esas intenciones, incluso aquí, cuando pretende alabar los estómagos y los paladares de varios nazis para luego poder mantener relaciones comerciales con ellos, no puede evitar dedicarle un piropo a la chica de uno de esos oficiales. Este rasgo de galán o conquistador — de donjuán, en una palabra—, parece haber sido una de las razones que empujaron a Spielberg a escoger a Neeson para el papel, dado que tiene una reputación similar a la del industrial nazi.

No debemos olvidar que, a pesar de todas sus conquistas, Schindler es un hombre casado. En la película este hecho no se descubre hasta que la señora Schindler llama a la puerta de la casa del simpatizante nazi, que es abierta por una de las amantes de éste. Su esposa, Emilie, le pone como única condición para seguir juntos que solo a ella la puedan llamar señora Schindler. No llegamos a oír la respuesta de Oskar, ya que el siguiente plano muestra a éste despidiendo a Emilie en la estación de tren, señal inequívoca de que Oskar ha respondido negativamente.

Con Emilie fuera de escena, Oskar sigue manteniendo relaciones con otras mujeres, y se halla con una de ellas cuando ve el desalojo del gueto por parte de soldados nazis, con todo el horror que ello lleva consigo. La mujer le dice, cuando ya han visto bastante, de irse, pero Oskar aún mantiene su mirada unos instantes más, esa mirada que sigue una de las pocas imágenes en color de la película, la de una niña que logra escapar momentáneamente de las manos de los soldados. El desarrollo de la película da a entender que a partir de ese instante Schindler toma una decisión aún más comprometida. Ese compromiso se puede observar en una escena anterior, en la que se contempla una imagen impropia de una película dirigida por Steven Spielberg: la de Schindler haciendo el amor con una de sus amantes. Lo que se ve es muy poco, lo suficiente para divisar que hay dos personas desnudas en la misma cama, y que cambian de posición. Además esto se utiliza para dar idea, una vez más, de la verdadera causa de Schindler, ya que la pareja es interrumpida mientras hacen el amor para que Oskar vaya a salvar a su contable, Itzhak Stern (Ben Kingsley), al que han metido en un tren con destino a cualquier campo de concentración por no llevar su tarjeta de identidad consigo.

Hay otro personaje, además de Schindler, que también muestra un afán, en este caso desmedido, por las mujeres. Se trata de Amon Goeth (Ralph Fiennes), el oficial nazi a quien Oskar compra, literalmente, sus judíos. Gran parte de la película se dedica a mostrar las similitudes y diferencias entre ambos hombres, dando a entender lo fina que es la raya divisoria que separa el bien del mal ${ }^{6}$. Esto lo vemos en su relación con las mujeres, pues, al igual, que Schindler, Goeth también disfruta de una amante. Su mayor pasión, sin embargo, se dirige hacia su sirvienta, Helen Hirsch (Embeth Davidtz), a la que desea pero no se atreve a poseer, dada su condición de judía. Como no puede ver saciado su deseo opta por pegarle, como se puede ver en la espléndida escena en el sótano, réplica de otra anterior que Schindler ha tenido

\footnotetext{
${ }^{6}$ Para una exploración a fondo de las connotaciones de ambos personajes, véase Díaz-Cuesta (2005-2008).
} 
Steven Spielberg - Sexualidad - Erotismo - Encuentros en la tercera fase - El color púrpura - La lista de Schindler

también con Hirsch, pero que en aquella ocasión terminaba con un casto beso y no con la brutal paliza con que finaliza la secuencia con Goeth.

Finalmente, Oskar vuelve a los brazos de su mujer, quien le ayudará a asistir a los judíos de la fábrica. Esta relación del matrimonio Schindler aparece en todo momento asexuada y se parece más a la que se puede encontrar entre dos hermanos. Con ello parece que se quiere decir que los esposos se concentran en los cuidados a sus judíos con una atención casi santificadora.

\section{DISCUSIÓN}

En la primera de las películas estudiadas el tema de las relaciones sentimentales, de la sexualidad y el erotismo, constituye una trama paralela del film que contribuye al desarrollo de la principal, mientras que en la segunda se sitúa en el centro de la narración, y en la tercera de ellas se utiliza como un rasgo diferenciador en el proceso de caracterización de los personajes.

En todos los casos, el tratamiento que Spielberg le da tanto a la sexualidad como el erotismo es muy respetuoso con los personajes y con el público al que van dirigidas las imágenes que ha creado.

Se observa, no obstante, un grado de evolución en el atrevimiento - o la necesidad, personal o debida a la trama- de mostrar escenas más explícitas en lo que se refiere a su contenido sexual.

En Close Encounters of the Third Kind se limita a besos de un marido con una mujer que no es su esposa (estos detalles se sitúan más en el plano de la moralidad que de la sexualidad, pero también han de ser tenidos en cuenta): la película recibió la calificación $P G$ (parental guidance) en Estados Unidos.

The Color Purple no llega a mostrar un plano en el que se vea la realización del acto sexual, pero sí se observan planos parciales de su consumación, así como la aproximación sexual, muy delicadamente tratada, entre dos mujeres: el film fue considerado por edades como $P G-13^{7}$ (parental guidance suggested for children under 13).

\footnotetext{
${ }^{7}$ Fue el propio Spielberg quien en su día promovió que existiera esta clasificación en concreto, la de PG-13. El director manifestaba en Vanity Fear. "The story of that was, I had come under criticism, personal criticism, for both Temple of Doom and, you know, Gremlins, in the same year. I remember calling Jack Valenti [then the president of the Motion Picture Association] and suggesting to him that we need a rating between $R$ and $P G$, because so many films were falling into a netherworld, you know, of unfairness. Unfair that certain kids were exposed to Jaws, but also unfair that certain films were restricted, that kids who were 13,14, 15 should be allowed to see. I suggested, "Let's call it PG-13 or PG-14, depending on how you want to design the slide rule," and Jack came back to me and said, "We've determined that PG-13 would be the right age for that temperature of movie." So I've always been very proud that I had something to do with that rating" (Windolf, 2008).
} 
Schindler's List se atreve con planos casi de cuerpo entero de Oskar Schindler con su amante. La película fue calificada con una $R$ (Restricted), pero no creo que la explicitud sexual haya sido lo que más haya pesado en esa catalogación, sino la mostración de violencia indiscriminada por parte de los nazis, únicamente atenuada por el uso del blanco y negro en la mayoría del texto fílmico.

Por tanto, la que pudiera habérsenos antojado peregrina idea, ésta de buscar huellas de sexualidad y erotismo en la obra de Spielberg, no resulta en modo alguno descabellada. Antes bien, el análisis de las tres obras que aquí hemos comentado invita a ampliar nuestro corpus hasta incluir la totalidad de obras del director, conscientes de que muy posiblemente en el resto de sus películas también encontraremos referencias al erotismo y a la sexualidad.

\section{REFERENCIAS}

BAXTER, John (1996): Steven Spielberg. The Unauthorised Biography. Harper Collins Publishers. Londres.

BUCKLAND, Warren (2006): Directed by Steven Spielberg: Poetics of the Contemporary Blockbuster. Continuum. Nueva York y Londres.

CALDEVILLA DOMÍNGUEZ, David (2005): El sello Spielberg. Editorial Vision Net. Madrid.

DÍAZ-CUESTA, José (1999): "El Color Púrpura: Alice Walker, Steven Spielberg, John Ford" en Revista de la SEECI, 3. Pág. 30-53. Consultado el 8 de octubre, 2013. Disponible en http://www.seeci.net/revista/hemeroteca/Numeros/Numero\%203/DiazCues.pdf.

DÍAZ-CUESTA, José (2003): "Un análisis de la adaptación al cine de La lista de Schindler por parte de Steven Spielberg", en Revista de la SEECI, 10. Pág. 30-52. Consultado el 8 de octubre, 2013. Disponible en http://www.seeci.net/revista/hemeroteca/Numeros/Numero\%2010/Cuesta.pdf

DÍAZ-CUESTA, José (2005-2008): "Man as Rescuer and Monster in Steven Spielberg's Film Text Schindler's List" en Journal of English Studies, 5-6. Pág. 63-81. Consultado el 8 de octubre, 2013. Disponible en http://publicaciones.unirioja.es/ojs2.4.2/index.php/jes/article/view/121/101

DÍAZ-CUESTA, José (2010): Hombres de Steven Spielberg. Un análisis de las representaciones de masculinidades en los textos fílmicos Duel, Jaws, Jurassic Park, The Lost World: Jurassic Park $y$ War of the Worlds. Servicio de Publicaciones de la Universidad de La Rioja. Logroño.

DÍAZ-CUESTA, José (2012): "Los hombres de Steven Spielberg en sus películas de monstruos", en Estudios sobre el Mensaje Periodístico, vol. 18, nº especial octubre. 
Steven Spielberg - Sexualidad - Erotismo - Encuentros en la tercera fase - El color púrpura - La lista de Schindler

Pág. 273-281. Consultado el 8 de octubre, 2013. Disponible en

http://revistas.ucm.es/index.php/ESMP/article/view/40981/39232

DIGBY, Joan (1993): "From Walker to Spielberg: Transformations of The Color Purple" en REYNOLDS, Peter (ed.): Novel Images: Literature in Performance (pp. 157-174). Routledge. Londres.

FRIEDMAN, Lester D. (2006): Citizen Spielberg. University of Illinois Press. Urbana y Chicago.

GORDON, Andrew M. (2008): Empire of Dreams: The Science Fiction and Fantasy Films of Steven Spielberg. Rowman \& Littlefield Publisher. Lanham, Maryland.

KENEALLY, Thomas (1993) [1982]: Schindler's List. Londres. Hodder and Stoughton. [Publicado originalmente como Schindler's Ark].

KOWALSKI, Dean A. (ed.) (2008): Steven Spielberg and Philosophy: We're Gonna Need a Bigger Book. The University Press of Kentucky. Kentucky.

KUBRICK, Stanley (dir.) (1968): 2001: A Space Odyssey. Metro-Goldwyn-Meyer y Stanley Kubrick Productions. EEUU y Reino Unido. [Título traducido en España como 2001: Una odisea en el espacio].

LOSHITZKY, Yosefa (ed.) (1997): Spielberg's Holocaust: Critical Perspectives on Schindler's List. Indiana University Press. Indiana.

MORRIS, Nigel (2007): The Cinema of Steven Spielberg. Empire of Light. Wallflower Press. Londres.

SÁNCHEZ-ESCALONILLA, Antonio (1995): Steven Spielberg. Royal Books. Barcelona.

SÁNCHEZ-ESCALONILLA, Antonio (2004): Steven Spielberg: Entre Ulises y Peter Pan. Cie Dossat 2000. Madrid.

SILET, Charles L. P. (2002): The Films of Steven Spielberg: Critical Essays. Scarecrow Press. Lanham, Maryland y Oxford.

SPIELBERG, Steven (dir.) (1977): Close Encounters of the Third Kind. Columbia Pictures Corporation en asociación con EMI Films. EEUU y Reino Unido. [Título traducido en España como Encuentros en la tercera fase].

SPIELBERG, Steven (dir.) (1985): The Color Purple. Amblin Entertainment, The GuberPeters Company y Warner Bros. Pictures. EEUU. [Título traducido en España como El color púrpura].

SPIELBERG, Steven (dir.) (1993): Schindler's List. Amblin Entertainment y Universal Pictures. EEUU. [Título traducido en España como La lista de Schindler]. 
WALKER, Alice (1992) [1982]: The Color Purple. (Special anniversary edition). The Women's Press Limited. Londres. [Publicada originalmente en 1982 por Harcourt Brace Jovanovich, Nueva York.

WASSER, Frederick (2010): Steven Spielberg's America. Polity Press. Cambridge y Malden, Massachusetts.

WINDOLF, Jim (2008): "Steven Spielberg" en Vanity Fair, 2 de enero. Consultado el 8 de octubre, 2013. Disponible en:

http://www.vanityfair.com/culture/features/2008/02/spielberg_qanda200802?printable $=$ true\&currentPage $=$ all\%5D

\section{José Díaz-Cuesta}

Profesor Contratado Doctor del área de Filología Inglesa en la Universidad de La Rioja (España), y Responsable de la Unidad de Audiovisuales de la Facultad de Letras y de la Educación de esta institución. Su investigación se centra en el estudio de las representaciones de masculinidades en textos literarios y fílmicos de autores anglonorteamericanos, así como en las relaciones que se establecen entre ambos tipos de textos. Es autor de la monografía Hombres de Steven Spielberg (2010), y ha editado, junto con Paula Requeijo Rey, el libro Innovar en el EEES a través de la investigación (2012). 